\title{
Medizinische Loci communes
}

\section{Formen und Funktionen einer ärztlichen Aufzeichnungspraxis im 16. und 17. Jahrhundert}

\author{
Michael Stolberg
}

\begin{abstract}
Physicians' Loci communes. Forms and Functions of Medical Commonplacing in the Sixteenth and Seventeenth Centuries

Commonplacing was one of the most widely practiced types of paper technology in the early modern period. Yet its place and function in medicine remain largely unexplored. Based on about two dozen manuscripts from the sixteenth and seventeenth centuries in which physicians used commonplacing to record excerpts from their reading as well as personal observations and ideas, this paper offers a first survey of the roles, forms and epistemic effects of medical commonplacing in the early modern period. Three principal types of commonplacing are identified, namely the systematic, the alphabetical and the sequential. The advantages and disadvantages of each type with regard to economy of space and time, flexibility, and order are pointed out. Among the potential epistemic effects of collecting and ordering information under "heads" are, in particular, a heightened awareness of contradictions between authors or observations on a specific topic, an appreciation for snippets of information as fact-like elements of knowledge inviting rearrangement, and a certain shift towards the notion of diseases as distinct entities endowed with specific, empirically observable properties.
\end{abstract}

Keywords: Commonplacing, paper technology, early modern medicine, historical epistemology, disease entities

Schlüsselwörter: Loci communes, paper technology, frühneuzeitliche Medizin, historische Epistemologie, Krankheitseinheiten

Die Anfertigung wohlgeordneter Exzerpte und Notizen gehörte unter den Gebildeten der Frühen Neuzeit zu den grundlegenden Kulturtechniken. Schon Schüler wurden darin unterrichtet. Detaillierte Anleitungen wie die von Francesco Sacchini (1570-1625), Jeremias Drexel (1581-1638) und Vincent Placcius (1642-1699) erschienen im Druck (Sacchini 1614, Drexel 1638, Placcius 1689, Cevolini 2006). Solche Aufzeichnungs- und Exzerpierpraktiken und ihre Bedeutung für das frühneuzeitliche information management haben in der jüngeren historischen Forschung einige Aufmerksamkeit gefunden (Meinel 1995, Zedelmaier/Mulsow 2001, Blair 2003, 2004a, 2004b, 2010a, 2010b). Das Interesse an der Kulturgeschichte des Lesens hat sich hier fruchtbar mit neueren, im weiteren Sinne praxeologischen Ansätzen in der 
Wissenschaftsgeschichte verbunden. Allerdings stützt sich die Forschung bislang fast ausschließlich auf die Analyse gedruckter Werke wie Jean Bodins Universae naturae theatrum oder Theodor Zwingers Theatrum vitae humanae, aus denen man in Ann Blairs Worten mittelbare Aufschlüsse „about the methods of note taking from which they were composed" $\mathrm{zu}$ gewinnen sucht (Blair 2004b: 95). Insbesondere für den Bereich von Naturgeschichte und Medizin sind handschriftliche Aufzeichnungspraktiken dagegen bislang kaum systematisch untersucht worden.

$\mathrm{Zu}$ den am weitesten verbreiteten, in gebildeten Kreisen allgemein bekannten und verwendeten Aufzeichnungspraktiken zählte die Anfertigung von Loci communes (Blair 1992, Yeo 2004: 4-9, Moss 2011). ${ }^{1}$ Ihr grundlegendes Merkmal ist die Strukturierung von Lektürefrüchten und anderen Notizen nach „Orten“ (lateinisch loci, griechisch topoi). Ursprünglich, in der aristotelischen Tradition der Topik, hatten die Loci ihren Platz in der Logik und Rhetorik (Goyet 1996: 614-621, Hotson 2007). Sie waren die „Orte“ oder "Sitze“ (sedes) der Argumente, derer sich ein Redner bedienen konnte. Manche Loci oder Sedes waren übergreifender, logisch-systematischer Natur, wie beispielsweise Genus, Species oder Proprium. Sie konnten Reden oder Abhandlungen über jeden beliebigen Gegenstand Struktur und Stringenz verleihen und waren insofern "gemeinsame" Orte, Loci communes. In der Renaissance bezog sich der Begriff jedoch zunehmend auf konkrete, thematisch definierte Wissensinhalte. Sie wurden $\mathrm{zu}$ Überschriften oder Schlagworten, was sich im Englischen bis heute im Wort topic für „Thema“ niederschlägt. Zwar sprach man weiterhin von Loci communes, aber je nach Wissensgebiet konnten nun ganz unterschiedliche Loci, im Sinne von inhaltlich definierten Kategorien gewählt werden (Ong 1958: 92-130, Schmidt-Biggemann 1983). Loci und Loci communes wurde zunehmend synonym gebraucht.

Im Zeitalter des Humanismus erlebten die Loci communes eine Blütezeit. Man verstand darunter vor allem Sammlungen von bekannten Sentenzen, Zitaten und Definitionen anerkannter, meist klassischer Autoren, die jeweils bestimmten Begriffen, Schlagworten oder eben „Orten“ zugeordnet wurden. Der heutige Begriff des Topos und die meist abwertende deutsche Übersetzung von Locus communis als "Gemeinplatz" geht auf diese Praxis der Sammlung von mehr oder weniger bekannten Wendungen der antiken Dichter und Philosophen zurück. Solche Loci communes anzufertigen, empfahlen humanistische Pädagogen zur Schulung des eleganten Ausdrucks und als unerschöpfliche Quelle von Copia, von inhaltlicher und sprachlicher Fülle für das eigene Schreiben und Reden (Erasmus 1514).

Loci communes ließen sich jedoch auch für andere Wissensbereiche als Poesie, Philosophie und Historie fruchtbar machen. Insbesondere in Naturgeschichte und Medizin machte die Akzentverlagerung von allgemeinen, abstrakten Kategorien hin $\mathrm{zu}$ konkreteren, mehr oder weniger 
fachspezifischen Inhalten die Loci communes auch für historisches Wissen im weiten, die Naturgeschichte einschließenden, zeitgenössischen Sinne offen, also für die Empirie (Schmidt-Biggemann 1983: 22 f.). Indem Erinnernswertes nach Schlagwörtern verzeichnet wurde, eröffnete diese Technik für das gesamte Spektrum der Disziplinen eine wichtige Alternative zum schlichten Notat, damals häufig Adversaria genannt, bei dem Wissenswertes weitgehend unstrukturiert in der Reihenfolge notiert wurde, in der es dem Verfasser bei der Lektüre oder in anderen Quellen begegnete. ${ }^{2}$ Was man in Theologie, Jurisprudenz oder Medizin über einen bestimmten Gegenstand las oder persönlich in Erfahrung brachte, konnte in Loci communes unter Schlagwörtern wie Miraculum, Donum oder Febres zusammengeführt werden. So entstand ein Wissensschatz, auf den man für Gespräche, Disputationen oder Veröffentlichungen zurückgreifen konnte, der aber auch für konkrete praktische Zwecke von Nutzen war. In seinem Hercules medicus empfahl Wolfgang Höfer dem Arzt in diesem Sinne, Aufzeichnungswürdiges, nach Loci communes geordnet, in einem gesonderten Buch zu notieren. Es könne ihm dann in schwierigen Krankheitsfällen, wenn Diagnose, Prognose oder die richtige Behandlung im Dunklen lägen, als Compendium oder als Promptuarium, also wörtlich als eine Vorratskammer dienen, der er mit scharfem Urteil das jeweils Passende entnehmen könne, um es am Patienten anwenden (Höfer 1675: Vorwort). Für den Arzt, der damals Patienten häufig in deren Häusern besuchte, konnten gut geordnete Loci communes die Rolle eines leicht transportablen, an die persönlichen Bedürfnisse angepassten Nachschlagewerkes erfüllen.

\section{Gedruckte Loci communes}

Im 16. und 17. Jahrhundert erschienen Loci communes $\mathrm{zu}$ verschiedenen Wissensfeldern im Druck. ${ }^{3}$ Das Genre war nicht völlig neu. Ähnliches boten schon die großen enzyklopädischen Kompilationen des Mittelalters wie Vincent de Beauvais' Speculum maius (Blair 2004a: 424). Doch das Formenspektrum und die thematischen Schwerpunkte veränderten sich jetzt. In der Theologie machte vor allem Philipp Melanchthon dieses Genre populär. In seinen Loci communes rerum theologicarum von 1521 wich er von der vertrauten Praxis ab, Sätze oder Passagen aus einem Buch der Heiligen Schrift der Reihe nach zu kommentieren. Stattdessen führte Melanchthon Stellen aus verschiedenen Büchern der Bibel unter etwa zwei Dutzend Oberbegriffen wie "Schöpfung“, „Menschen“, „Laster“, „Strafen“, „Glaube“ und „Hoffnung" zusammen (Melanchthon 1521). In der Jurisprudenz ließen sich in ähnlicher Weise Lesefrüchte und sonstiges Wissenswertes zu einem bestimmten Sachverhalt oder Tatbestand aus unterschiedlichen Rechtsquellen zusammenführen. ${ }^{4}$ 
Auch ärztliche Autoren brachten gedruckte Loci communes heraus. Diese sind historisch bislang kaum untersucht. Otto Brunfels' Theses seu communes loci totius rei medicae (Brunfels 1532) waren nach zeitgenössischem Sprachgebrauch eher Lemmata (Drexel 1638: 85 f.). Ohne nähere Angaben zum Inhalt führten sie wie eine moderne Sachbibliographie einschlägige Literaturstellen aus den Werken verschiedener medizinischer Autoren unter Schlagwörtern zusammen. Diese Tradition führten im ausgehenden 16. Jahrhundert Pascal Lecoq in seiner Biblioteca medica und Israel Spachius in seinem Nomenclator scriptorum medicorum fort, der im Untertitel ausdrücklich eine Ordnung „nach den Loci communes der Medizin“ ankündigte (Lecocq 1590, Spachius 1591). Andere Autoren boten dagegen eine umfassende inhaltliche Darstellung. Sie konnten dabei auf älteren Darstellungsformen aufbauen, die beispielweise in der Krankheitslehre dem alten Schema „von Kopf bis Fuß“ folgten und selbst eine alphabetische Anordnung nach Krankheitsbegriffen findet sich auch schon in älteren Werken zur medizinischen Praxis. ${ }^{5}$ Das ehrgeizigste Unterfangen dieser Art waren Francesco Valleriolas Loci medicinae communes, tribus libris digesti, die auf rund 1500 Folioseiten die gesamte damalige Heilkunst abbilden wollten. ${ }^{6}$ Auf eine in Loci communes geordnete Darstellung der vielfach verstreuten Auffassungen und Beobachtungen in den Werken Galens beschränkte sich bescheidener Aloysius Mundella (1568). Vorwiegend auf die Bedürfnisse des praktizierenden Arztes zielte wiederum Wolfgang Höfer (1775) mit seinem bereits erwähnten Hercules medicus, sive locorum communium liber.

\section{Handschriftliche Loci communes}

Sind gedruckte medizinische Loci communes bislang kaum untersucht, so gilt das erst recht für die handschriftlichen Loci communes frühneuzeitlicher Ärzte. ${ }^{7}$ Gedruckte Loci communes dürften in der Regel aus handschriftlichen Auszeichnungen hervorgegangen sein, die ihre Verfasser nach dem Prinzip der Loci communes angelegt hatten (Blair 1992: 543-548) und sie mögen ihrerseits wiederum anderen als Vorbild für persönliche Aufzeichnungen gedient haben. Gedruckte Loci communes - in der Medizin wie in anderen Disziplinen unterscheiden sich jedoch in einem zentralen Punkt ganz grundlegend von jenen Loci communes, die zeitgenössische Gelehrte handschriftlich für den persönlichen Gebrauch anfertigten. Gedruckte Loci communes boten ein abgeschlossenes Werk, in dem ein vorweg bekannter Stoff in entsprechende Anordnung gebracht wurde. ${ }^{8}$ Handschriftliche Loci communes zeichneten sich dagegen gerade durch ihre Offenheit aus. Sieht man vom Sonderfall jener handschriftlichen Loci communes ab, die erst sekundär durch die saubere und gegebenenfalls strukturierende Abschrift oder Übertragung von ungeordneten 
Aufzeichnungen entstanden, so waren handschriftliche Loci communes ein Arbeitsinstrument, das sich erst im Laufe der Arbeit füllte und dessen Inhalt zu Beginn allenfalls in Umrissen absehbar war. Anders als Valleriolas umfangreiches Werk zielten sie in der Regel auch nicht auf die vollständige, umfassende Darstellung eines Wissensfelds oder Gegenstands. Vielmehr erlaubten sie es dem Verfasser, mehr oder weniger selektiv nur das zu notieren, was ihm vor dem Hintergrund seines Wissens und seiner Interessen erinnerungswert oder im Hinblick auf eine mögliche zukünftige Verwendung nützlich erschien. Gedruckte Loci communes boten für solche auf die persönlichen Interessen und Bedürfnisse zugeschnittenen Aufzeichnungen keinen Ersatz, und entsprechend bescheiden blieb ihre Zahl, gerade in der Medizin.

Die Anfertigung handschriftlicher Loci communes war allem Anschein nach auch unter Medizinstudenten und Ärzten weit verbreitet. In den seltenen Fällen, in denen handschriftliche Nachlässe von einzelnen Ärzten in größerem Umfang überliefert sind, finden sich regelmäßig Loci communes. Manche Mediziner - das bekannteste Beispiel ist der Arzt und Philosoph John Locke haben sogar eine ganze Reihe davon hinterlassen. Die Anfertigung von medizinischen Loci communes wurde in Anleitungen für das Medizinstudium und die ärztliche Praxis ausdrücklich empfohlen (Kijper 1643, Hoffmann 1738: 35 f.), und vermutlich geben diese ihrerseits einen Rat wieder, der den Studenten auch mündlich von ihren Professoren auf den Weg gegeben wurde. Thomas Bartholin pries ihre Vorzüge und empfahl den Berufsanfängern, den Tyrones, ein Compendium novorum titulorum zu führen, indem sie für jede Disziplin einen Band nähmen, in den sie alles, was aufzeichnungswürdig sei, an seinem rechten Ort (suo loco) kurz (brevissime) vermerkten. ${ }^{9}$ Nicht zuletzt bei gelehrten Disputationen, in opponendo vel respondendo, waren Loci communes dem Rigaer Arzt Piper zufolge überaus nützlich. ${ }^{10}$ Auch in der kurzen Zusammenfassung von Jeremias Drexels ausführlicher Exzerpieranleitung, die der schlesische Arzt Martin Kerger 1695 veröffentlichte, spielte die Technik der Loci eine zentrale Rolle (Kerger 1695, vergleiche Drexel 1638).

Die studierten Ärzte der Frühen Neuzeit waren über weite Strecken ähnlich ausgebildet und sozialisiert wie andere Gelehrte. Sie hatten in der Regel etliche Jahre an einer Lateinschule verbracht und anschließend ein Studium der Artes absolviert, ehe sie sich speziell der Medizin zuwandten. Somit waren sie mit der Technik der Loci communes bestens vertraut. Der unbekannte Verfasser eines umfangreichen, in St. Gallen überlieferten Bandes von medizinischen Loci communes setzte diesen sogar mehrere Seiten mit Exzerpten zur Bedeutung und Technik der Loci communes voran, die er den Werken von Melanchthon, Viktorin Strigel, Johannes Sturm und Joachim Camerarius dem Älteren entnahm. ${ }^{11}$ Es überrascht daher nicht, dass auch aus ärztlicher Feder Loci communes überliefert sind, die ganz nach humanistischem Vorbild erinnernswerte Passagen, Zitate und Definitionen von 
antiken Dichtern und Philosophen unter verschiedenen Überschriften verzeichneten. ${ }^{12}$

Ähnlich wie die gedruckten medizinischen Loci communes changierten handschriftliche Loci communes zwischen Bibliographie und inhaltlichen Notizen. Zitate und konkrete Aussagen stehen zuweilen neben bloßen Hinweisen auf die Seite in einem bestimmten Werk, in dem Näheres dazu stehe. So finden sich in den Loci communes des Zerbster Arztes und späteren Marburger Professors Johannes Magirus (1615-1697) neben zahlreichen inhaltlich gefüllten Ausführungen auch Stellen wie die zu Hydrops vesicalis, wo er sich auf den Eintrag „Eugal[enus] f. 152 usque ad f. 158“ und den Hinweis auf die „Historia curatae mulieris“ im gleichen Werk sowie auf die kurze Notiz „Senn[ert] 1[iber] 3 pr[acticae] f. 644“ beschränkt. ${ }^{13}$ Vermutlich folgten die Verfasser hier einem Rat, der sich auch in einschlägigen Anleitungen findet, sich nämlich bei Büchern, die man selbst besaß und gegebenenfalls erneut konsultieren konnte, mit kurzen Notizen zu begnügen (Kijper 1643: 266 f.) Für Magirus lässt sich der Besitz mancher der erwähnten Bücher tatsächlich anhand eines Katalogs der nachgelassenen Bücher bestätigen. ${ }^{14}$

Private Loci communes hatten für ihre Verfasser und Besitzer offenbar erheblichen Wert. Als sich der junge Christoph Hellwig besorgt bei Johann von Muralt nach dem Verbleib seiner Habseligkeiten erkundigte, die er offenbar in Leiden zurückgelassen hatte, bat er ihn an erster Stelle nach seinen Sennert-Büchern sowie nach Locos meos communes philologicos und seinen Locos physicos zu suchen. ${ }^{15}$ Und seine bald darauf geäußerte Bitte, Muralt möge herausfinden, wo und an wen die Witwe Hottingers ${ }^{16}$ seine Loci communes verkauft habe, legt nahe, dass derlei handschriftliche Aufzeichnungen auch einen gewissen Marktwert besaßen. ${ }^{17}$ Johannes Placotomus regte gar die gemeinschaftliche Anfertigung von medizinischen Loci communes an. Er sei dabei, die gesamte Medizin nach einzelnen Gebieten zu ordnen, schrieb er Ende 1545 an den anhaltisch-dessauischen Leibarzt Wolfgang Furmann. Man könne, was man bei den einzelnen Autoren finde, jeweils an der richtigen Stelle eintragen, erklärte er, und forderte Furmann auf, auch selbst entsprechende Stellen zu sammeln und zu übersenden. ${ }^{18}$ Petrus Johannes Wiburg bat 1602 Sigismund Schnitzer, er möge ihm seine Locos communes zur Wirksamkeit von Theriak und Bezoar mitteilen, den er, Wiburg, erfolgreich bei einem Buben eingesetzt habe, nachdem er von Wiburgs rasendem wenn nicht sogar tollwütigen Hund gebissen worden sei. ${ }^{19}$

In den Archiven und Bibliotheken finden sich handschriftliche Loci communes dennoch nur in bescheidener Zahl. Das dürfte jedoch in erster Linie der generell sehr bruchstückhaften Überlieferung solcher privater Aufzeichnungen geschuldet sein. Ganz Ähnliches gilt beispielsweise auch für die handschriftlichen Vorlesungsmitschriften, die damals wohl so gut wie jeder Student anfertigte. Abgesehen von den medizinischen und chemischen Loci communes im umfangreichen Nachlass von John Locke (Meynell 1993, Milton 
1987, 1997, Yeo 2004) sind handschriftliche medizinische Loci communes meines Wissens bislang nie näher untersucht worden. Der vorliegende Beitrag gründet auf der Untersuchung von etwa zwei Dutzend frühneuzeitlicher handschriftlicher Loci communes aus ärztlicher Feder, auf die ich bei meinen vielfältigen Recherchen zu Briefen, Praxisjournalen und anderen handschriftlichen Dokumenten von Ärzten in deutschsprachigen Archiven und Bibliotheken im Lauf der Jahre gestoßen bin. Sie stammen aus dem deutschen und holländischen Sprachraum und sind fast ausschließlich auf Latein verfasst. Manche sind - teilweise schon auf dem Buchrücken - ausdrücklich mit der Bezeichnung Loci communes versehen, bei anderen wandten die Verfasser die Loci-communes-Technik an, gaben ihren Aufzeichnungen jedoch Bezeichnungen wie Mnemoneutikon oder Notata medica. In Umfang, Form und Aufmachung gibt es große Unterschiede. Das Spektrum reicht von einer einzigen - ausdrücklich mit Loci communes betitelten - Seite ${ }^{20}$ bis hin $\mathrm{zu}$ kostspielig in Leder gebundenen Foliobänden mit Hunderten von Seiten. ${ }^{21} \mathrm{Sie}$ waren ganz offensichtlich nur für den privaten Gebrauch bestimmt. Einzige Ausnahme ist das Promptuarium sive loci communes latinitatis aus der Feder des böhmischen Arztes Georg Handsch, ${ }^{22}$ ein rund 1.200 Seiten starker, nicht primär medizinisch, sondern enzyklopädisch angelegter Band, der offenbar für den Druck vorbereitet, aber nie veröffentlicht wurde. ${ }^{23}$

\section{Typologie}

Die Bezeichnung Loci communes verweist bei genauerer Betrachtung auf verschiedene Formen der Anordnung. Ich möchte im Folgenden eine Einteilung in drei grundlegende Typen vorschlagen, den systematischen, den alphabetischen und den sequentiellen. Diese Typologie ist aus meiner Analyse medizinischer und naturgeschichtlicher Loci communes gewonnen, dürfte aber auch auf Loci communes zu anderen Disziplinen oder Wissensbereichen übertragbar sein. Wie wir sehen werden, erfüllten diese drei Grundtypen in je unterschiedlicher Weise die vier zentralen, aber teilweise widersprüchlichen Anforderungen an nützliche Exzerpier- und Aufzeichnungstechniken: Zeitökonomie, Platzökonomie, Ordnung und Flexibilität.

Im ersten, systematischen Typ von Loci communes waren die Eintragungen nach inhaltlichen Gesichtspunkten gegliedert (Abb. 1). Die Reihenfolge der Schlagwörter ähnelte der Anordnung von Kapitelüberschriften in einem gedruckten Buch und die Schlagworte oder Capita waren zuweilen sogar einem verbreiteten Standardwerk entlehnt. ${ }^{24}$ Auf den ersten Blick ähnelten solche Aufzeichnungen damit einem durch Überschriften sauber gegliederten Exzerpt aus einem Buch. Sie konnten beispielsweise mit Gott, Kosmos, Erde und den Elementen beginnen, ${ }^{25}$ oder konkreter mit dem Wesen der Medizin 


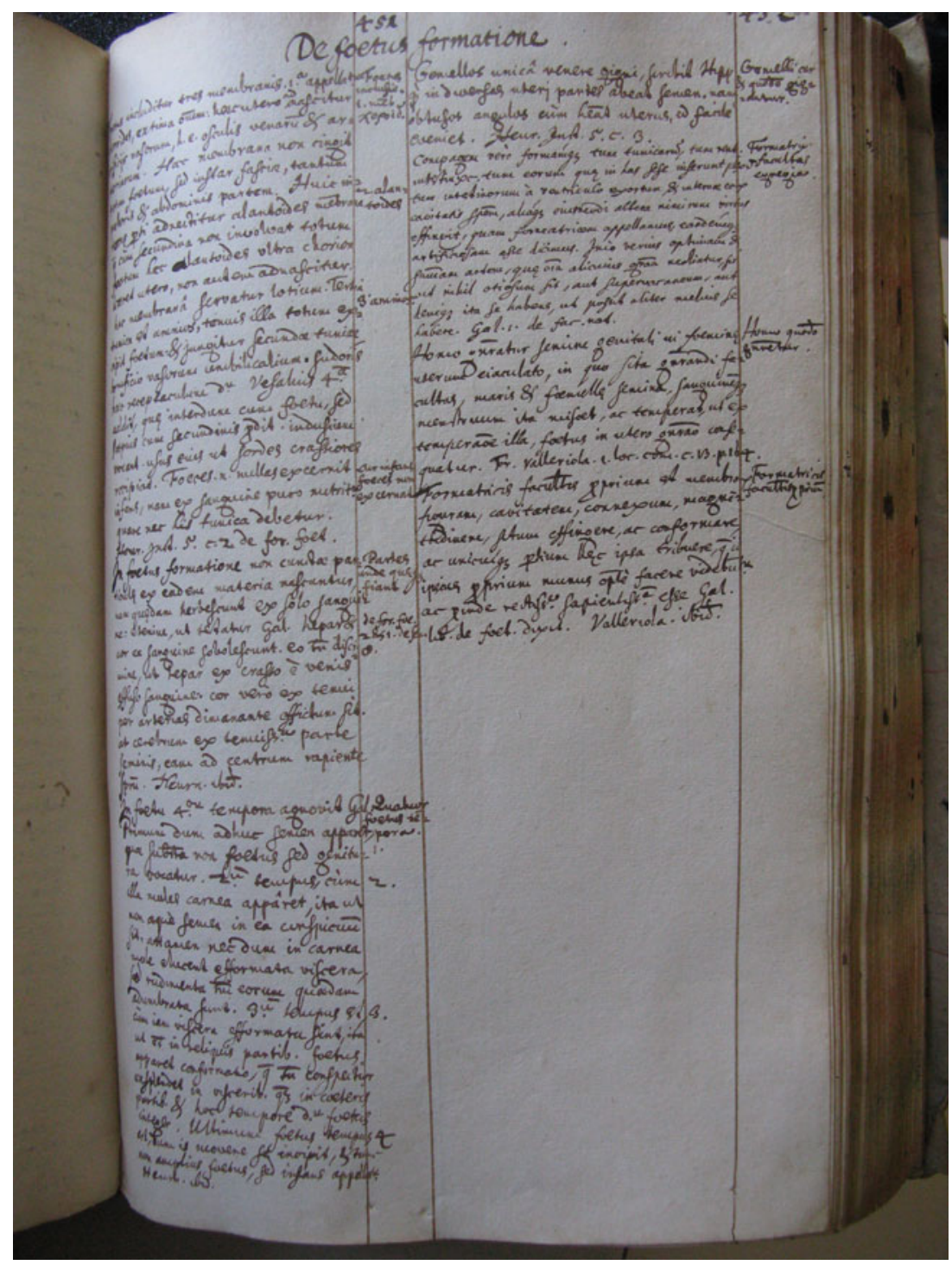

Abb. 1 Medizinische Loci communes eines unbekannten Verfassers um 1600 vom Typ I: systematische Anordnung (Mit freundlicher Genehmigung der Kantonsbibliothek St. Gallen) $^{26}$

und den Res naturales und Res non naturales, gefolgt von Eintragungen zu einzelnen Krankheitsbegriffen. ${ }^{27}$ Der entscheidende Unterschied zu einem gewöhnlichen Exzerpt war, dass unter ein und derselben Überschrift in der Folgezeit weitere Exzerpte aus anderen Stellen des gleichen Werks oder auch aus ganz anderen Werken verzeichnet wurden. So finden sich beispielsweise in den oben erwähnten St. Gallener Loci communes unter der Überschrift De febribus et cal[ore] p[raeter] n[aturali] in g[e]n[e]re rund 20 einschlägige 
Äußerungen und Zitate aus Werken von Galen, Hippokrates, Avicenna, Emanuel Stupanus, Leonhard Fuchs, Jean Fernel und Girolamo Mercuriale sowie eine mündliche Äußerung, die Caspar Bauhin in einer Prüfung gemacht hatte. $^{28}$

Die St. Gallener Handschrift steht allerdings zugleich auch stellvertretend für ein Phänomen, das in ähnlicher Weise bei der Untersuchung von anderen Loci-communes-Handschriften dieses systematischen Typs ins Auge fällt. Gut gefüllten Seiten stehen in dieser Handschrift zahlreiche andere gegenüber, die kaum Einträge enthalten oder abgesehen von der Überschrift völlig leer blieben. Insbesondere im zweiten, den lokalisierten Krankheiten von Kopf bis Fuß gewidmeten Teil des Foliobandes, mit den Spalten 626-1142, ist dies der Fall. Die meisten Überschriften blieben ohne Eintrag und nur bei ganz vereinzelten Krankheiten findet sich beispielsweise der Hinweis auf eine einschlägige Observatio Pieter van Foreests oder gar auf die persönliche Beobachtung an einem „hiesigen Bürger“ ${ }^{29}$ Offensichtlich begannen die Verfasser von loci dieses Typs zwar nicht selten mit großem Enthusiasmus. Sie ließen sich für teures Geld einen mehr oder weniger umfangreichen, nicht selten aufwendig gebundenen Band mit Blankoseiten herstellen oder kauften einen solchen, und trugen in sauberer Schrift die einzelnen Schlagwörter oder Capita ein. Doch früher oder später gaben sie auf. Auch das - vermutlich in frühen Jahren begonnene - Mnemoneutikon der medizinischen Praxis von Joachim Camerarius dem Jüngeren (1534-1598) - sein Vater, ein bekannter Humanist, hatte unter anderem philosophische Loci communes publiziert (Camerarius 1552) - enthält unter der Mehrzahl der sauber angeordneten Überschriften keinerlei Einträge, oder allenfalls einen kurzen Hinweis, beispielsweise auf ein bestimmtes Rezept. ${ }^{30}$ Nicht viel besser gefüllt ist das Memoriale practicum von Erasmus Reinhold dem Jüngeren (1538-1592). Auch hier stehen voll beschriebene Seiten, die bestimmten Medikamententypen wie den Purgantia oder einzelnen Krankheiten wie der Epilepsie gewidmet waren, anderen gegenüber, die ohne jeden Eintrag blieben. ${ }^{31}$ Manche Loci communes dieses Typs haben offenbar überhaupt nur deshalb überlebt, weil ihre Besitzer die leeren Blätter später für ganz andere, unsystematische Aufzeichnungen verwendeten. $^{32}$

Rückblickend ist dieses Scheitern verständlich. Zum einen war die Platzökonomie bei diesem Typ eine große Herausforderung. Es war kaum abzuschätzen, wie viel Raum die einzelnen Loci oder Capita jeweils benötigen würden, denn diese waren von sehr unterschiedlichem Gewicht. Der Verfasser konnte annehmen, dass beispielsweise Einträge zu Affectus animi oder Melancholia mehr Raum beanspruchen würden als jene für Tactus oder Lac, aber möglicherweise stieß er wider Erwarten auf eine Fülle von erinnerungswerten Belegen für die relative Bedeutung von Tastsinn und Sehsinn oder die Vorzüge des Stillens. Georg Handsch skizzierte vor diesem Hintergrund ein zweistufiges, damit freilich zwangsläufig auch sehr viel zeitraubenderes 
Verfahren: Er verfasste Loci communes gegründet auf vorgängige ungeordnete Notizen und Vorlesungsmitschriften und verschaffte sich zunächst einen Überblick über die verwendeten Überschriften und deren Raumbedarf. Wenn er diese Farrago, dieses Durcheinander abschreiben wolle, so notierte er sich, müsse er zunächst auf Papier die nötigen loci in der richtigen Ordnung auflisten. Dann müsse er diese Farrago durchsehen und schauen, „wo viel und wo wenig an irgendeinem Locus sein wird" und dies auf dem Papier entsprechend vermerken. Schließlich müsse er die Schlagwörter, jeweils den ermittelten Platz lassend, vom Papier in ein Buch übertragen, und anschließend die ungeordneten Notizen entsprechend abschreiben. ${ }^{33}$ Hier wurden die Loci communes also zu einer Aufzeichnungspraxis zweiter Ordnung.

Der vermutlich noch wichtigere Grund für das Scheitern dieses Typus war jedoch der beschränkte Nutzen. Die Wahl der Schlagwörter und erst recht die thematische Anordnung nach der Art eines Lehrbuchs zielte tendenziell auf Vollständigkeit, auf das gesamte medizinische Wissen der Zeit. Eine Dispositio in breves ac succinctas theses totius medicinae theoricae, also eine Anordnung der gesamten theoretischen Medizin in kurzen und treffenden Lehrsätzen, nahm sich Isaac Habrecht (1589-1633) mit seiner Sammlung von Exzerpten aus den Werken von Fernel, Fuchs, Heurne, Riolan und anderen vor. ${ }^{34}$ Zumindest Generalia in omni praxi medicinae observanda, also, was allgemein in jeder medizinischen Praxis zu beachten war, wollte Erasmus Reinhold verzeichnen. ${ }^{35}$ Das musste Stückwerk bleiben. Hätte der Schreiber jedesmal, wenn er beispielsweise auf eine Aussage über die Natur, Diagnose oder Behandlung von Fieber stieß, einen Eintrag machen wollen, so hätte das einen extrem hohen Zeitaufwand erfordert. Dieses Wissen war aber in einer ganz ähnlichen Anordnung über gedruckte Institutiones und andere zusammenfassende medizinische Lehrwerke der Zeit schon recht bequem zugänglich. Auch sie stellten üblicherweise auch abweichende Auffassungen oder Theorien vor und diskutierten sie. Es ist insofern kein Zufall, dass dieser Typ von Loci communes, soweit sich die Handschriften datieren lassen, offenbar in erster Linie von Studenten gepflegt wurde. Habrecht etwa war gerade 17 Jahre alt als er seine Loci communes begann, Erasmus Reinhold war 22 und Medizinstudent im vierten Jahr. Hier stand wohl noch ganz das Bemühen im Vordergrund, sich das kanonische Grundwissen anzueignen und dem Gedächtnis einzuverleiben.

Der zweite, alphabetische Typus von Loci communes bot deutlich mehr Flexibilität und Raum für eine individuelle, persönliche Gestaltung (Abb. 2). Hier waren die Schlagwörter wie in einem Lexikon von A bis Z angeordnet. Manche Schreiber versahen zu diesem Zweck ein Blankobuch vorweg mit den erwarteten Schlagworten oder Überschriften in alphabetischer Reihenfolge. Dieses Verfahren eignete sich vor allem dann, wenn das Spektrum der voraussichtlich benötigten Schlagwörter recht klar war. Namen und Anzahl der unterschiedlichen Darreichungsformen von Arzneien beispielsweise waren 


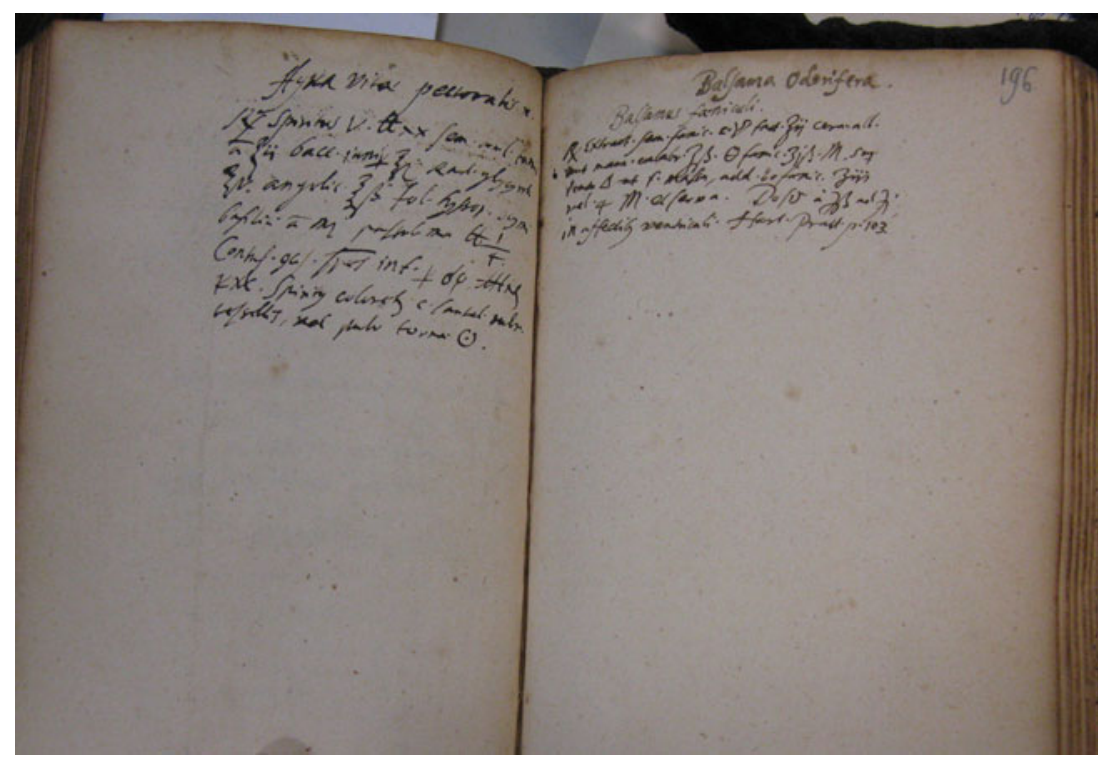

Abb. 2 Loci communes von Laurenz Blumentrost um 1650 vom Typ II: alphabetische Anordnung (Mit freundlicher Genehmigung der Universitätsbibliothek Marburg) ${ }^{36}$

begrenzt und einigermaßen vorhersehbar. In seinen 1649 begonnenen medizinisch-pharmazeutischen Loci communes konnte Laurenz Blumentrost daher unter dem Buchstaben M jeweils eine Seite für Einträge zu Magisteria, Mortificatoria und Morselli beziehungsweise Morsuli vorsehen, gefolgt von Seiten für Einträge zu Olea per expressionem, Olea per infusionem et coctionem und Potiones purgantes. ${ }^{37}$

Alternativ, das war die zweite Möglichkeit, konnten sich die Schreiber darauf beschränken, jeweils eine bestimmte Zahl von Seiten für die einzelnen Buchstaben des Alphabets zu reservieren, indem sie vorweg entsprechend Buchstaben, A, B und so weiter auf die noch leeren Seiten schrieben, ${ }^{38}$ oder indem sie die Seiten zunächst ganz leer ließen und bei neuen Schlagwörtern jeweils abzuschätzen versuchten, wo diese innerhalb einer alphabetischen Anordnung ihren Platz finden würden. Hier konnten die Schlagwörter also im Laufe der Arbeit nach Bedarf gewählt und eingetragen werden. So versah ein unbekannter Leser von Paracelsus' Astronomia magna - möglicherweise der Berliner Paracelsist und brandenburgische Leibarzt Leonhard Thurneisser einen rund 50 leere Blätter umfassenden Band zunächst mit großen Lettern von A bis Z, um dann unter den passenden Buchstaben kurze Hinweise oder Sentenzen einzutragen, etwa unter $C$ „Das Corpus ist gewesen der Anfang aller Ding“ oder unter F „Das Firmament ein leiblichs Wessen hatt. “39 Auch John Locke experimentierte in frühen Jahren mit diesem Typus. ${ }^{40}$

Der grundsätzliche Nachteil der alphabetischen Anordnung war, das musste offenbar auch Locke feststellen, dass hier, ähnlich wie im ersten, 
systematischen Typ, oft viele Seiten leer blieben, und zwar keineswegs nur, weil der Schreiber das Interesse verlor. ${ }^{41}$ Über kurz oder lang gab es unter einem bestimmten Buchstaben beziehungsweise an einer bestimmten Stelle des Alphabets keine leeren Seiten mehr für weitere Einträge beziehungsweise um ein benötigtes neues Schlagwort zu bilden. Dann musste entweder improvisiert werden, beispielsweise mit mehreren Schlagworten auf der gleichen Seite oder durch die Nutzung leer gebliebener Seiten am Schluss. Oder aber ein neues Loci-communes-Buch musste begonnen werden, obwohl in dem bislang benutzten bei manchen Buchstaben womöglich noch viele Seiten weitgehend leer waren. Es überrascht insofern nicht, dass sich auch bei diesem zweiten, alphabetischen Typus das Phänomen einer späteren Umnutzung findet. So verwandte Caspar Weckerlin den leer gebliebenen Platz seiner alphabetisch angeordneten Loci communes, um dort 1616 diverse Fälle aus seiner Praxis, Verschreibungen und Schuldforderungen zu notieren, die mit den ursprünglichen Capita wie Mala, Medium, Medicina, Morbus und Motus nichts zu tun hatten. ${ }^{42}$

Am flexibelsten und zugleich platzsparendsten war der dritte, sequentielle Typ von ärztlichen Loci communes, der hier vorgestellt werden soll (Abb. 3). Kerger empfahl ihn in seiner Exzerpieranleitung ausdrücklich (Kerger 1695: $\$ 10)$. Der Verfasser machte hier seine Einträge mehr oder weniger der Reihe nach, so wie sie ihm unterkamen. Er gab weder Schlagwörter noch eine

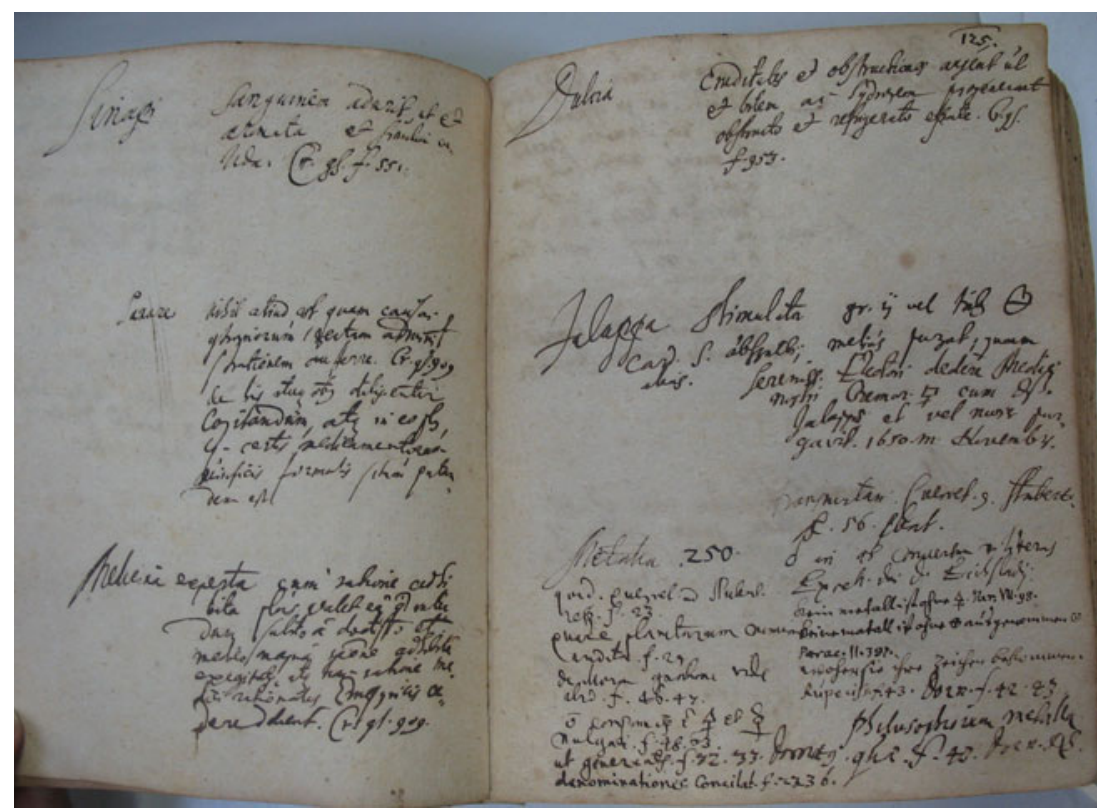

Abb. 3 Loci communes von Johannes Magirus um 1650 vom Typ III: sequentielle, indexbasierte Anordnung (Mit freundlicher Genehmigung der Universitätsbibliothek Marburg) ${ }^{43}$ 
bestimmte Anordnung vor und bot damit besonders große Spielräume für eine individuelle, persönliche Gestaltung, in der sich die jeweiligen Interessen, Lektüren und Erfahrungen des Verfassers unmittelbar niederschlugen.

Dieser Typus steht damit den Notata oder Adversaria nahe, also Notizbüchern, in die Erinnerungswertes ungeordnet, einfach der Reihe nach verzeichnet wurde. ${ }^{44}$ Allerdings gab es zwei entscheidende Unterschiede. Zum einen wurde jede Aufzeichnung mit einem möglichst prägnanten Schlagwort versehen oder diesem zugeordnet, für das manche Schreiber eine eigene Spalte reservierten und das andere zumindest durch eine größere oder dickere Schrift oder eine andersfarbige Tinte kenntlich gemacht. Es ist diese explizite thematische Strukturierung, die klare und in der Regel deutlich hervorgehobene Zuordnung der Einträge zu einem bestimmten Schlagwort oder Locus, die auch diese Aufzeichnungsform zu Loci communes macht. Zum anderen wurde das „Chao[s]“ der Einträge, wie Kerger (1695: \$ 10) es formulierte, in aller Regel mit Hilfe dieser Schlagwörter über einen alphabetischen Index erschlossen, der es erlaubte, Aufzeichnungen zu einem bestimmten Gegenstand noch rascher wiederzufinden. Blair (2010b: 91) hat zwar erstaunt angemerkt, dass in den von ihr untersuchten Quellen, ungeachtet Drexels einschlägiger Empfehlung von 1638, vor dem ausgehenden 17. Jahrhundert niemanden seine Aufzeichnungen mit einem Index versehen habe. Die hier untersuchten Handschriften ergeben jedoch ein anderes Bild. So finden sich schon in den diversen Notizbüchern von Georg Handsch aus der Mitte des 16. Jahrhunderts sehr ausführliche Indizes, und auch die Loci communes von Johannes Magirus aus der Mitte des 17. Jahrhunderts sind mit einem Index versehen. Drexels Empfehlung griff offenbar eine etablierte Praxis auf.

Dieser dritte Typus von Loci communes war von vornherein nicht auf Vollständigkeit angelegt. Bot sich der systematische Typ vorwiegend für Studenten an, die größere, womöglich ähnlich strukturierte Werke unter einer beschränkten Zahl von Überschriften exzerpieren wollten, so eignete sich der sequentielle Typ eher für den belesenen oder erfahrenen Arzt und Naturphilosophen, der zu einer unabsehbaren Vielzahl von Themen nur das kurz eintragen wollte, was ihm wirklich bemerkenswert und erinnerungswert erschien. ${ }^{45}$ Der Nachteil der sequentiellen Eintragung war allerdings, dass sich Eintragungen zum gleichen Schlagwort nun an verschiedenen Stellen des Bandes fanden, an denen der Verfasser dieses Schlagwort jeweils neu vergab. Zwar konnte er versuchen, unter den einzelnen Einträgen jeweils noch etwas Platz für weitere zu lassen, aber je mehr Platz er ließ, desto mehr riskierte er wiederum, dass dieser letztlich ungenutzt blieb, weil sich doch keine weiteren Einträge für das betreffende Schlagwort fanden, ehe die letzte Seite beschrieben war. Trug der Verfasser Aufzeichnungen zum gleichen Schlagwort aber auf verschiedenen Seiten ein, so ließen sich diese nicht mehr auf einem Blick erfassen. Sie bildeten nur eine virtuelle Einheit, die erst über den Index - oder gar durch simples Durchblättern - konstituiert werden musste. So waren 
beispielsweise in den Loci communes des Johannes Magirus die Einträge zum Schlagwort Aurum auf sieben, teilweise weit auseinander liegende Seiten verteilt - und dabei sind weitere Seiten mit Einträgen zu spezielleren Begriffen wie Aurum potabile noch nicht mitgezählt. ${ }^{46}$

\section{Epistemische Effekte}

Loci communes erfüllten, wie andere Exzerpier- oder Aufschreibepraktiken, in hervorragender Weise mnemotechnische Funktionen. Schon der Akt des Schreibens, so betonten zeitgenössische Exzerpieranleitungen, half das Geschriebene im Gedächtnis zu verankern. Durch wiederholte Lektüre der Aufzeichnungen konnte es sodann dauerhafter eingeprägt werden. Er wolle seine Eintragungen am Ende jedes Tages, jede Woche sowie alle drei Monate durchlesen, nahm sich in diesem Sinne der junge Georg Handsch vor, so wie das auch seine Lehrer und Kollegen machten. ${ }^{47}$ Darüber hinaus konnte Gelesenes und/oder Erfahrenes mit Hilfe von Loci communes für die zukünftige Verwendung verfügbar gehalten werden. Zielten die humanistischen Loci communes primär auf das Ideal der copia, der Fülle an Zitaten und Wendungen, auf die man in Veröffentlichungen, in Reden, in Briefen oder im gelehrten Gespräch zurückgreifen konnte (Erasmus 1514, vergleiche Goyet 1996), so versammelten medizinische Loci communes insbesondere Wissen, dem der Verfasser für die ärztliche Praxis, die allägliche Behandlung der Patienten, besondere Bedeutung zumaß, das ihm aber andererseits auch nicht selbstverständlich schien.

Loci communes sind jedoch weit mehr als nur passive Spiegel von Rezeptionsvorgängen. Als disziplinenübergreifend angewandte und an die einzelnen Disziplinen adaptierte Technik hatten sie das Potential, das Denken und die forschende Praxis maßgeblich zu beeinflussen und neue Erkenntnisse und Theorien hervorzubringen (Blair 2004b: 90). Für die hier untersuchten Loci communes frühneuzeitlicher Ärzte möchte ich insbesondere drei zentrale epistemische Wirkpotentiale hervorheben, die sich schlagwortartig unter den Begriffen Pluralisierung, Kategorisierung und Dekontextualisierung zusammenfassen lassen.

Indem Loci communes die einschlägigen Definitionen, Theorien, Meinungen oder Befunde verschiedener Autoren oder in den Werken ein und desselben Autors zu einem bestimmten Gegenstand unter einem Schlagwort versammelten, führten sie Unterschiede zwischen den Autoren und Widersprüche im Werk einzelner Autoren unmittelbar vor Augen, machten sie im systematischen und alphabetischen Typ sogar auf einen Blick erfassbar. So sammelte der Arzt und Kalendermacher Johannes Magirus in seinen Loci communes unter dem Schlagwort Annus Stellen aus den Werken von 
Hippocrates, Galen, Paulus von Aegina und Sethus Calvisius, aus denen sich eine Jahreslänge von 350, 354 und 365 Tagen ergab. ${ }^{48}$ Insofern gedruckte Werke ihrerseits allem Anschein nach häufig auf solchen handschriftlichen, in Form von Loci communes geführten Aufzeichnungen ihrer Autoren beruhten, konnten diese durch die je unterschiedliche Auswahl und Anordnung von Stellen beispielsweise aus dem umfangreichen Werk von Galen in eine ganz unterschiedliche (Re)Konstruktion von dessen Lehren münden (Maclean 2002: 229 f.). Auf diese Weise trug die Loci-communes-Technik dazu bei, das Bewusstsein für die plurale Vielfalt der zeitgenössischen Medizin und die Widersprüche innerhalb ihres theoretischen Lehrgebäudes, und selbst innerhalb des Werks führender Autoritäten, zu schärfen. Sie wirkte damit in eine ähnliche Richtung wie die damals verbreiteten Abrisse widersprüchlicher Lehrmeinungen in den medizinischen Lehrbüchern, deren Autoren sich vermutlich wiederum auf solche persönlichen Aufzeichnungen stützten. Sie half, antike und zeitgenössische Autoritäten in Frage zu stellen und förderte eine gewisse Skepsis gegen absolute Erklärungsansprüche.

Ein zentrales distinguierendes Charakteristikum von Loci communes war zweitens ihre Ordnungsfunktion. Im Gegensatz zu gewöhnlichen Notizbüchern, in die „tumultarie“, wie Albert Kijper (1614-1655) es formulierte (Kijper 1643: 265), alles Mögliche durcheinander verzeichnet wurde, kategorisierten Loci communes das Material thematisch, nach Schlagwörtern. Die Loci-communes-Technik spiegelte damit auf ihre Weise eine damals gerade in der Medizin sehr verbreitete Wertschätzung für eine „rationale“ Methode. ${ }^{49}$ Jeder wisse, so hob Wolfgang Höfer (1675: Praefatio) in seinem Hercules medicus, sive locorum communium liber in diesem Sinne einleitend hervor, wie wichtig es für den Gelehrten sei, eine Ordnung oder eine gewisse Methode zu beachten.

Die Auswahl der zu verzeichnenden Stellen und ihre Zuordnung zu bestimmten Schlagwörtern war jedoch stets auch ein kreativer, potentiell wissensproduktiver Akt. ${ }^{50}$ Er setzte in der Regel die Entscheidung für eines von mehreren vorgegebenen Schlagworten oder gar die Formulierung eines neuen Schlagwortes voraus. Kerger empfahl dementsprechend große Sorgfalt „in titulo fabriciendo": Das Schlagwort solle den zu exzerpierenden Kern des Ganzen umfassen. ${ }^{51}$ In der Bewertung dessen, was den Kern des Ganzen ausmachte, und in der Wahl des treffenden Locus kamen jedoch zwangsläufig persönliche Vorannahmen und Präferenzen zum Ausdruck.

Gerade in der Medizin und Naturphilosophie war dieses kreative Element der Loci-communes-Technik sehr ausgeprägt. Denn medizinische und naturphilosophische Loci communes beschränkten sich in ihren Aufzeichnungen nicht auf bloße Lesefrüchte. Ihre Verfasser trugen auch empirische Beobachtungen zu einzelnen Kranken und praktische Erfahrungen mit bestimmten Arzneien oder Erlebnisse im Umgang mit Patienten, Angehörigen und anderen Heilkundigen in ihre Loci communes ein, eigene und von anderen 
mitgeteilte (Blair 1992). Die Zuordnung von empirischen Beobachtungen und persönlichen Erfahrungen, ihre Subsummierung unter bestimmte Oberbegriffe war diesen jedoch nicht inhärent. Auch sie spiegelte bestimmte Vorannahmen, Sichtweisen und Präferenzen. Für die medizinischen Loci communes ist in diesem Zusammenhang die Zuordnung von einzelnen Beobachtungen $\mathrm{zu}$ bestimmten Krankheitsbegriffen wie „Skorbut", „Krebs“, „Dreitagesfieber“, „Hysterie“, „Wassersucht" von besonderem Interesse. Ausdrücklich empfahl Daniel Gerhard Morhof (1692: 157) in seiner Exzerpieranleitung speziell dem praktisch tätigen Arzt, dessen erste Aufgabe die Heilung von Krankheiten sei, eine solche Gliederung seiner Collectanea nach Krankheitsbegriffen. Krankheiten wie "Skorbut“ oder „Krebs“ sind jedoch bekanntlich nicht natürlich gegeben. Ihre Abgrenzung beruht letztlich auf der Abstraktion aus der Beobachtung von einzelnen Menschen mit mehr oder weniger unterschiedlichen Beschwerden, Empfindungen, körperlichen Veränderungen oder Verhaltensweisen - und diese Abstraktion kann im Kulturund Epochenvergleich sehr verschiedene Zuordnungen hervorbringen. Autoren, die unterschiedliche Textstellen und Beobachtungen an einzelnen Patienten unter einem solchen Krankheitsbegriff als Locus communis versammelten, verliehen diesem eine spezifische Gestalt und Bedeutung. Sie machten aus persönlichen Beobachtungen und überlieferten Geschichten von individuellen Krankheitsfällen, von denen keiner dem anderen völlig glich, eine mehr oder weniger klar umschriebene Krankheitseinheit und definierten, welche Krankheitsphänomene sich dieser zuordnen ließen und welche nicht. Wenn beispielsweise der Ulmer Arzt Johannes Frank in seiner nach Krankheiten geordneten, handschriftlichen Sammlung von Observationes aus der eigenen Praxis Patienten mit Unterschenkelgeschwüren dem Krankheitsbild „Skorbut" zuordnete, so gründete das auf einer spezifischen, damals verbreiteten Deutung des Skorbuts als Folge einer Ansammlung scharfer Säfte im Körper, die die Hautoberfläche gewissermaßen zerfraßen. ${ }^{52}$ Heute würden viele dieser Kranken nicht als „skorbutisch“ diagnostiziert. Zuweilen fassten frühneuzeitliche Krankheitsbegriffe denn auch Symptome zu Krankheiten wie Pica, Vapeurs oder Suffocatio uteri zusammen, die wir in dieser Form heute gar nicht mehr kennen (Stolberg 2003: 215-229, Zedlitz 2012).

Nach Krankheitsbegriffen geordnete oder zumindest mit entsprechenden Krankheitsbegriffen überschriebene medizinische Observationes oder Consilia, die einzelne Krankengeschichten entsprechend versammelten, erschienen - offenbar gegründet auf die handschriftliche Aufzeichnung von Fällen aus der eigenen ärztlichen Praxis - auch in wachsender Zahl im Druck und erreichten eine breitere Öffentlichkeit (Stolberg 2007, Pomata 2010, 2011). Die Nähe solcher thematisch, nach Krankheitsdiagnosen geordneter Observationes und Consilia zu den Loci communes war auch Zeitgenossen durchaus bewusst. Mit Nachdruck pries Theodor Zwinger in diesem Sinne die großen Verdienste, die sich Johann Schenck von Gräfenberg für das medizinische Gemeinwesen, die 
Medica republica, erworben habe indem er die Observationes und Curationes, die sich verstreut bei verschiedenen Schriftstellern fänden, in Loci communes anordne. ${ }^{53}$ So wurde aus der privaten, individuellen Aufzeichnung von einzelnen Krankengeschichten und Behandlungserfolgen und ihrer Zuordnung zu bestimmten Krankheitsbegriffen letztlich ein kollektives Unterfangen. Ein wachsender Bestand an empirischen Beobachtungen entstand, der es in Verbindung mit der überlieferten Literatur erlaubte, die Natur der einzelnen Krankheiten immer genauer zu bestimmen, die Bandbreite der jeweiligen Krankheitsbilder einzugrenzen, womöglich unterschiedliche voneinander zu trennen, die Ursachen der einzelnen Krankheiten zu bestimmen und nicht zuletzt ihre bestmögliche Behandlung zu erkennen.

Indem die Loci communes Krankheitsbegriffe zu Capita, zu Schlagworten, machten, trieben sie zugleich eine der zentralen Entwicklungen in der frühneuzeitlichen Medizin insgesamt voran, die sich auch in anderen Texten zur medizinischen Praxis verfolgen lässt, nämlich die Durchsetzung eines neuen Krankheitsverständnisses: Krankheiten wurden immer mehr zu abgrenzbaren Wesenheiten mit einer typischen Erscheinungsform und die Identifizierung und Benennung der Krankheit geriet zur zentralen diagnostischen Aufgabe. In der Forschungsliteratur hält sich zwar hartnäckig die Auffassung, Krankheiten seien bis zum 18. Jahrhundert oder sogar noch länger auf eine Störung des Gleichwichtes der vier natürlichen Säfte (Blut, gelbe und schwarze Galle sowie Schleim) und/oder ihrer Qualitäten (warm, kalt, trocken, feucht) zurückgeführt worden. Dabei handelt es sich jedoch nur um einen liebgewonnenen Topos, der einer genaueren Prüfung nicht standhält. Die Idee eines je spezifischen Gleichgewichts der Säfte und Qualitäten blieb allenfalls im Temperament-Begriff lebendig und half physiologische (sowie charakterliche) Unterschiede zwischen den Menschen zu erklären. In der Krankheitslehre aber spielte das Gleichgewichtsmodell in Werken zur medizinischen Praxis wie in den zahllosen Observationes und Consilia spätestens seit der zweiten Hälfte des 16. Jahrhunderts kaum noch eine Rolle (und möglicherweise wird selbst seine Bedeutung in der mittelalterlichen Medizin überschätzt, die durchaus eine Reihe von Krankheitsbezeichnungen kannte). Die meisten Krankheiten wurden im 16. und 17. Jahrhundert vielmehr auf eine mehr oder weniger spezifische Krankheitsmaterie zurückgeführt, die sich allenfalls gelegentlich und in einem sehr weiten Sinne auch einmal auf ein lokales Säfte- oder Qualitätsungleichgewicht zurückführen ließ, beispielsweise auf eine überhitzte Leber, die das Geblüt „verbrannte“ und „scharfe“ Säfte hervorbrachte. Die Behandlung zielte folgerichtig in aller Regel nicht darauf, ein gestörtes Gleichgewicht der Säfte wieder herzustellen, sondern möglichst gezielt, die Krankheitsmaterie auszuführen oder unschädlich zu machen (Stolberg 2003).

Praxisaufzeichnungen und Consilia des 16. Jahrhunderts zeigen allerdings, dass die ärztliche Diagnose damals noch vorwiegend darauf zielte, die 
jeweiligen pathologischen Prozesse als solche aufzudecken und damit einer Behandlung zugänglich zu machen, beispielweise einen schwachen, verschleimten Magen, der zur Anhäufung von rohen, zähen Stoffen in den Gefäßen und schließlich zu einer Verstopfung der Milz führte. Der Name der Krankheit war sekundär. Im Laufe der Frühen Neuzeit wurde dagegen gerade diese Zuordnung des einzelnen Krankheitsfalls zu einer bestimmten Krankheitskategorie, die Benennung, immer wichtiger. Ärztliche Fallsammlungen wurden vermehrt - wenn auch nicht durchgehend - nach Krankheiten geordnet. Die Auffassung setzte sich durch, dass Menschen an der "gleichen“ Krankheit leiden konnten und jenseits aller ärztlichen Individualisierungsrhetorik mehr oder weniger gleich $\mathrm{zu}$ behandeln waren. Im 18. Jahrhundert brachte diese Sichtweise schließlich ausgefeilte nosologische Klassifikationsversuche hervor, in denen Krankheiten wie Pflanzen nach ihren typischen Merkmalen und Verwandtschaften systematisch geordnet wurden. ${ }^{54}$ Zweifellos hatte diese Entwicklung eine ganze Reihe von Ursachen, aber viel spricht dafür, dass die Loci-communes-Technik einen wichtigen Beitrag leistete, indem sie den Arzt dazu brachte, unterschiedliche Symptombilder und individuelle Krankheitsverläufe innerhalb einer gewissen Variationsbreite jeweils einem bestimmten Krankheitsbegriff zuzuordnen.

Ein dritter epistemischer Effekt medizinischer und naturphilosophischer Loci communes ist schwerer zu greifen, war jedoch langfristig vermutlich der wirkmächtigste: Als eine von Jugend auf geübte und praktizierte Kulturtechnik hat die verbreitete Praxis der Anfertigung von Loci communes einen wichtigen Platz in der Geschichte der wachsenden Wertschätzung für „wissenschaftliche Tatsachen“. Mit gutem Grund empfahl kein geringerer als Francis Bacon (1605: 106) nachdrücklich „the disposition and collocation of that knowledge which we preserve in writing" in Form eines "good digest of common places". Vor allem Lorraine Daston hat in den vergangenen Jahren die zentrale epistemologische Bedeutung der "Fakten“ in den modernen Wissenschaften als Ergebnis eines historischen Prozesses herausgearbeitet, in dem das 17. und 18. Jahrhundert eine Schlüsselrolle einnahmen. Ein zentrales Charakteristikum von Fakten - beziehungsweise ihrer Kommunikation - so ihr argumentativer Ausgangspunkt, ist deren Kürze. In dieser Kürze spiegelt sich ihr idealiter reiner, neutraler, voraussetzungs- und theorieloser Status. Denn derart konstituierte Fakten folgen keiner vorgegebenen Ordnung. Wie die Karten eines Kartenspiels können sie nach Belieben durchmischt und immer wieder in eine neue Anordnung gebracht werden, beispielsweise in Form von Tabellen oder Listen. Sie können immer wieder aufs Neue und auf ganz unterschiedliche Weise als Bausteine und empirische Belege für eine unbegrenzte Vielfalt von Argumenten, Theorien, Erklärungsmodellen oder Klassifikationen dienen (Daston 2001). 
Die Parallelen zu Wesen und Funktion von Loci communes - vor allem jener des alphabetischen und sequentiellen Typs - liegen auf der Hand. ${ }^{55}$ Charakteristisches Merkmal solcher Loci communes war die Dekontextualisierung. Die in den Loci communes verzeichneten, in der Regel sehr kurz und konzise zusammengefassten Textpassagen oder persönlichen Beobachtungen wurden aus ihrem ursprünglichen Zusammenhang gerissen und nach dem Ermessen des Verfassers einzelnen Schlagwörtern zugeordnet. Die verbreitete Praxis der Anfertigung von Loci communes war somit bestens geeignet, einen mentalen Habitus zu fördern, in dem kurze Fakten oder Erkenntnisschnipsel eine Schlüsselrolle spielten. So wie Schuljungen und Studenten der Artes lernten, kurze Zitate und Schlüsselaussagen aus den Werken der antiken Dichter, Philosophen nach Loci geordnet zu verzeichnen, so begannen sie als Ärzte und Naturforscher neben entsprechenden Exzerpten aus der einschlägigen Literatur auch kurze, prägnante Aussagen und Erkenntnisse aus dem Buch der Natur zu verzeichnen, ohne sie gleich in eine systematisches, durch eine bestimmte Theorie vorgegebenes Ordnungssystem zu pressen (Bacon 1623: 264 f.).

\section{Loci communes als ärztliche Aufzeichnungspraxis}

Handschriftliche, für den persönlichen Gebrauch angefertigte Loci communes, dies sollte dieser Beitrag hinreichend deutlich gemacht haben, sind ein lohnender und bislang zu Unrecht vernachlässigter Gegenstand medizin- und wissenschaftsgeschichtlicher Analyse. In mancher Hinsicht den späteren Laborjournalen vergleichbar, eröffnen sie wertvolle Einblicke in zeitgenössische Denk- und Ordnungsprozesse in Medizin und Naturgeschichte. Sie lassen die Themen, Beobachtungen und Debatten erkennen, die für die Verfasser von besonderem persönlichen Interesse waren. Sie belegen und veranschaulichen deren Bemühen, dem Gelernten und Beobachteten Sinn und Ordnung zu verleihen. Nicht zuletzt trug die Loci-Technik dazu bei, überkommene Wahrheitsansprüche in Frage zu stellen, Ordnungskategorien - wie die Einteilung der Krankheiten - zu verfestigen und einen mentalen Habitus zu fördern, in dem isolierte, dekontextualisierte Fakten zum neuen erkenntnistheoretischen Ideal werden konnten.

In welchem Ausmaß die Loci-communes-Technik solche epistemische Wirkungen auf die damalige Medizin und Naturgeschichte entfaltete und welchen Stellenwert sie hier im Vergleich zu anderen Aufzeichnungstechniken und Publikationsformen hatte, bleibt freilich durch zukünftige Forschungen noch genauer zu klären. Deren Aufgabe wird es auch sein, weitere Handschriften ausfindig zu machen und zu untersuchen, die hier vorgeschlagene Typologie gegebenenfalls $\mathrm{zu}$ verfeinern und die Besonderheiten und 
Gemeinsamkeiten handschriftlicher medizinischer Loci communes im Vergleich zu jenen aus anderen Disziplinen wie der Theologie und der Jurisprudenz herauszuarbeiten.

\section{Anmerkungen}

1 Sharpe (2007: 277) formuliert treffend: „During the Renaissance period, indeed perhaps from the twelfth century to the eighteenth century, the keeping of a commonplace book was a normal habit of reading." Siehe auch den Beitrag von Fabian Krämer in diesem Themenheft Paper Technologies. Eine Forschungstechnologie der frühneuzeitlichen Wissenschaft. NTM. Zeitschrift für die Geschichte der Wissenschaften, Technik und Medizin N. S., 21 (2013), im gleichen Heft.

2 Ein anschauliches Beispiel hierfür ist Zürich (Ms. Car. X 205, Adversaria von Salomon Hottinger (um 1676)), mit aneinandergereihten Exzerpten aus mehr als 40 Werken.

3 Zu den gedruckten Loci communes oder commonplace books der Frühen Neuzeit siehe die ausführliche Analyse bei Moss 1996.

4 Vgl. beispielsweise die fast 1200 Seiten umfassende juristische Enzyklopädie von Sole/ Schultes 1607.

5 So Ketham 1522: foll. XIXv-XXIIr, Tractatus septimus qui de egritudinibus particularibus inscribitur, secundum alphabeti ordinem dispositus.

6 Valleriola 1562, zit. Praefatio. Weitere Auflagen erschienen 1563, 1573 und 1589.

7 Moss (1996: VIII) hat in ihrem grundlegenden Werk zu den gedruckten Loci communes erklärtermaßen auf die Untersuchung von medizinischen und juristischen Loci communes verzichtet, weil diese "a complex contextualization best reserved for a separate study“ verdienten. Auch Blair beschränkt sich im Wesentlichen auf den Hinweis: „Medical note taking would also be worth studying" (Blair 2004b: 91, Fußnote).

8 Einen Sonderfall bilden die Loci communes des Johannes Foxus (Foxus [1557]). Der dicke Folioband bot Hunderte von ansonsten leeren Seiten, an deren Kopfende in alphabetischer Reihenfolge Schlagwörter aus dem gesamten Spektrum des Wissenswerten vorgegeben waren, unter denen der Leser seine persönlichen Aufzeichnungen eintragen sollte. 1572 und 1585 erschienen weitere Auflagen, was eine beachtliche Nachfrage vermuten lässt. In einem Exemplar, das in der Universitätsbibliothek Freiburg i. Br. (Abendländische, neuzeitliche Handschriften 305) überliefert ist, hat sich der unbekannte Verfasser im Wesentlichen auf medizinische Aufzeichnungen beschränkt.

9 Bartholin 1771: 149 (posthume Ausgabe mit einem Vorwort von Bartholin von 1672).

10 Königliche Bibliothek Kopenhagen, (Ms. Thottske S 4 689, Piper, Collegium patholog[icum] et terapiam [sic] ut et collegium medico politicum (mit großer Nähe zu Hoffmann 1738)).

11 Kantonsbibliothek St. Gallen (Ms. 408).

12 Staatsbibliothek zu Berlin - Preußischer Kulturbesitz (Ms. Lat. Qu. 41, Loci communes des späteren Arztes und Medizinprofessors Salomon Alberti); Österreichische Nationalbibliothek (Cod. 9607, foll. 1r-97v und foll. 107v-125v: poetische Loci communes von Georg Handsch), siehe auch: von einem ärztlichen Autor, Bruno Seidel, im Druck veröffentlicht (Seidel 1589).

13 Universitätsbibliothek Marburg (Ms. 97: fol. 23). Magirus ist Gegenstand eines DFGForschungsprojektsam Institut für Geschichte der Medizin der Universität Würzburg (http://www.medizingeschichte.uni-wuerzburg.de/aerztliche_praxis/projekt_stolberg.html, [zuletzt zugegriffen am 23. November 2012]).

14 Universitätsbibliothek Marburg (Ms. 664, Catalogus librorum ex Magiri Bibliotheca).

15 Zentralbibliothek Zürich (Ms. Z II 608a: 21-23, Brief Christoph Hellwigs an Johann von Muralt vom 18. Juni 1667)

16 Gemeint ist möglicherweise der Züricher Orientalist und Theologe Johann Heinrich Hottinger, der am 5. Juni 1667 bei einem Bootsunglück in der Limmat ertrank. Er hatte in Leiden studiert und kurz zuvor einen Ruf an diese Universität erhalten. 
17 Zentralbibliothek Zürich (Ms. Z II 608a: 27 f., Brief Hellwigs an Muralt vom 13. Juli 1667).

18 Landeshauptarchiv Sachsen-Anhalt, Abteilung Dessau (GAR. NS., Nr. 244: Brief von Johannes Placotomus an Wolfgang Furman, Datierung erschlossen). Den Hinweis auf diesen und den folgenden Brief verdanke ich den Mitarbeitern des Projekts „Frühneuzeitliche Ärztebriefe“ der Bayerischen Akademie der Wissenschaften am Institut für Geschichte der Medizin der Universität Würzburg.

19 Hornung [1626]: 393, Brief von Petrus Johannes Wiburg an Sigismund Schnitzer vom 26. Februar 1602.

20 Österreichische Nationalbibliothek (Cod. 22006: fol. 181r: Loci communes aphorismorum Hippocratis et aliorum libellorum et authorum aliquot).

21 Beispielsweise Universitätsbibliothek Leipzig (Ms. 2494, Loci communes eines unbekannten Verfassers, um 1600).

22 Handsch und sein Nachlass stehen im Mittelpunkt meiner aktuellen Forschungen; vgl. bislang Senfelder 1901 und Smolka/Vaculínová 2010.

23 Österreichische Nationalbibliothek (Cod. 9550 (1549)). Auf die Absicht der Veröffentlichung verweist neben der sehr sauberen, einheitlichen Form die direkte Anrede an den Lector (18r).

24 Siehe auch Blair 2010b: 90 f. zu dem Züricher Philologen J. C. Hagenbuch, der sogar das Layout eines gedruckten Werks übernahm.

25 Kantonsbibliothek St. Gallen (Ms. 408).

26 Ebd.: coll. $452 \mathrm{f}$.

27 Österreichische Nationalbibliothek (Cod. 11239: foll. 3r-16r: Loci communes in Aphorismos Hyppocratis von Georg Handsch).

28 Kantonsbibliothek St. Gallen (Ms. 408: coll. 600-602).

29 Ebd.: col. 1114 (De menstruorum suppressione) und coll. $1031 \mathrm{f}$ (De hydrope).

30 Universitätsbibliothek Erlangen (Ms. 935). Ähnliches gilt für Universitätsbibliothek Leipzig (Ms. 2494: Loci communes eines unbekannten Verfassers, um 1600).

31 Staatsbibliothek Bamberg, Bamberger Sammlung (Msc. misc. 385).

32 Universitätsbibliothek Marburg (Ms. 118, Laurenz Blumentrost, 1649 begonnen). Den Hinweis auf diese Handschrift verdanke ich Sabine Schlegelmilch (Würzburg). Vermutlich handelt es sich um Laurenz Blumentrost den Älteren (1619-1705), den späteren zaristischen Leibarzt, der 1648 in Jena zum Dr. med. promoviert wurde.

33 Österreichische Nationalbibliothek (Cod. 11239: fol. 100v).

34 Königliche Bibliothek Kopenhagen (Ms. Gl. Kongl. 4 1691, Farrago medica collecta von Isaac Habrecht, 1606).

35 Staatsbibliothek Bamberg, Bamberger Sammlung (Msc. misc. 385).

36 Universitätsbibliothek Marburg (Ms. 118: foll. 195v-196r).

37 Ebd.: foll. 253r-263r.

38 Ein weiteres Beispiel sind die - allerdings allgemein humanistischen - Loci communes des späteren Arztes und Medizinprofessors Salomon Alberti (Staatsbibliothek zu Berlin Preußischer Kulturbesitz (Ms. Lat. Qu. 41)).

39 Staatsbibliothek zu Berlin - Preußischer Kulturbesitz (Ms. germ. fol. 524).

40 Bodleian Library Oxford (Ms. Locke d.10, Lemmata ethica; Ms. Locke d.11, Lemmata physica).

41 Ein extremes Beispiel für ein rasch nachlassendes Interesse bietet Österreichische Nationalbibliothek (Cod. 11210 von Georg Handsch: foll. 73v-79v). Auf die sorgfältige ausgeführte Überschrift „Loci communes in aphorismos Hyppocratis“ folgen nur leere Seiten.

42 Königliche Bibliothek (Ms. Gl. kongl. $4^{\circ}$ 1694). Vermutlich war der Verfasser der spätere Strassburger Arzt gleichen Namens, der 1613 in Leiden promoviert wurde.

43 Universitätsbibliothek Marburg (Ms. 97, foll. 124 f).

44 Überblick bei Chatelain 1997. Der Begriff „Adversaria“ wurde damals allerdings unterschiedlich gebraucht und konnte auch allgemein Notizbücher oder selbst handschriftliche Anmerkungen in Büchern bezeichnen.

45 Eine damals noch kaum praktizierte Alternative waren Notate auf einzelnen, jeweils mit capita versehenen Zetteln, wie sie Joachim Jungius verwandte (Meinel 1995). Mit Hilfe eines Zettelkastens konnten die Notate wahlweise in eine alphabetisch oder systematische Anordnung gebracht werden. 
46 Universitätsbibliothek Marburg (Ms. 97).

47 Österreichische Nationalbibliothek (Cod. 11208).

48 Universitätsbibliothek Marburg (Ms. 97: fol. 450).

49 So forderte Fonseca (1596: 4) „prae caeteris exactissimam methodum, sine qua nihil recte vel scribi, vel operari potest".

50 Kevin Sharpe (2000: 191) hat die kreative Rolle des zuordnenden Schreibers treffend zusammengefasst: „as he selects, paraphrases, arranges, glosses, cross-references and indexes, he performs a very individual reading and interpretation".

51 Kerger 1695: $₫$ 8. Auf die Mühe, bestimmte Einträge unter mehreren Schlagworten zu verzeichnen, haben die Ärzte, zumindest in dem hier untersuchten Quellenmaterial, verzichtet.

52 Stadtbibliothek Ulm (Mss. Franc 8a und 8b).

53 Brief vom 19. August 1584 (in Schenck von Gräfenberg 1594: foll. 3v-4r).

54 Vgl. auch den Beitrag von Hess und Mendelsohn im Themenheft Paper Technologies. Eine Forschungstechnologie der frühneuzeitlichen Wissenschaft. NTM. Zeitschrift für die Geschichte der Wissenschaften, Technik und Medizin 21 (2013), Heft 1.

55 Daston (2004: 445) hat ausdrücklich auf diese Parallele hingewiesen: „the Renaissance humanist practice of excerpting short, pithy quotations from long texts for florilegia and commonplace books bears a close resemblance to the excerpting of short, pithy facts from the continuum of experience“. Ähnlich argumentierte auch schon Blair (1992: 545) in ihrer Untersuchung des (gedruckten) Universae naturae theatrum von Jean Bodin.

\section{Literatur}

Bacon, Francis, 1605. Two Bookes [...] of the Proficience and Advancement of Learning, Divine and Humane. London: Tomes.

Bartholin, Thomas, 1711. De libris legendis dissertationes, hg. von Joh. Gerh. Meuschen. Frankfurt a. M.: Wildt.

Blair, Ann, 1992. Humanist Methods in Natural Philosophy. The Commonplace Book. Journal of the History of Ideas, 53, 541-551

Blair, Ann, 2003. Reading Strategies for Coping with Information Overload, ca. 1550-1700. Journal of the History of Ideas, 64, 11-28.

Blair, Ann, 2004a. Focus: Scientific Readers. An Early Modernist's Perspective. Isis, 95, 420-430.

Blair, Ann, 2004b. Note Taking as an Art of Transmission. Critical Inquiry, 31, 85-107.

Blair, Ann, 2010a. The Rise of Note-Taking in Early Modern Europe. Intellectual History Review, 20, 303-316.

Blair, Ann, 2010b. Too Much to Know. Managing Scholarly Information Before the Modern Age. New Haven/London: Yale University Press.

Brunfels, Otto, 1532. Theses seu communes loci totius rei medicae. Strassburg: Andianus.

Camerarius, Joachim, 1552. Arithmologia ethica, loci communes, et epigrammata. Leipzig: Valentinus Papa.

Cevolini, Alberto, 2006. De arte excerpendi. Imparare a dimenticare nella modernità. Florenz: Olschki

Chatelain, Jean-François, 2007. Les recueils d'adversaria aux XVIe et XVIIe siècles. Des pratiques de la lecture savante au style de l'érudition. In: Frédéric Barbier, Hg., Le livre et l'historien. Études offertes en l'honneur du Professeur Henri-Jean Martin. Genf: Librairie Droz, 169-186.

Daston, Lorraine, 2001. Perché i fatti sono brevi? Quaderni storici, 108, 745-770.

Daston, Lorraine, 2004. Taking Note(s). Isis, 95, 443-448.

Drexel, Jeremias 1638. Aurifodina artium et scientiarum omnium excerpendi solertia. München: Leysser.

Erasmus, Desiderius, 1514. De duplici copia verborum ac rerum commentarii duo. Paris: [ohne Druckervermerk]. 
Fonseca, Rodericus a, 1596. Opusculum, quo adolescentes ad medicinam facile capessendam instruuntur, casus omnium febrium methodice discutiuntur, \& curantur. Florenz: apud Michaelangelum Sermattellium.

Foxus, Johannes, [1557]. Locorum communium tituli et ordines centum quinquaginta, ad seriem praedicamentorum decem descripti. Basel: Oporinus.

Goyet, Francis, 1996. Le sublime du "lieu commun". L'invention rhétorique dans l'Antiquité et à la Renaissance. Paris: Champier.

Höfer, Wolfgang, 1675, Hercules medicus, sive locorum communium liber: in quo plerorumque humani corporis affectuum curationes attinguntur, et quicquid in iis vel theorico, vel practico consideratione dignum, compendiose pertractatur. Nürnberg: Endter.

Hoffmann, Friedrich, 1738. Medicus politicus sive regulae prudentiae. Leiden: Bonk.

Hornung, Johannes, Hg. [1626]. Cista medica, qua in epistolae clarissimorum Germaniae medicorum, familiares, et in re medica, tam quoad hermetica et chymica, quam etiam galenica principia, lectu jucundae et utiles, cum diu reconditis experimentis asservantur. Nürnberg: Halbmayr.

Hotson, Howard, 2007. Commonplace Learning. Ramism and its German Ramifications, $1543-1630$.

Kerger, Martin, 1695. Methodus excerpendi, Drexeliana succinctior. Zusammen mit: Drexel, Jeremias, Aurifodina artium et scientiarum omnium solertia. Breslau: Müller

Ketham, Johannes de, 1522. Fasciculus medicine. Venedig: Arrivabenus.

Kijper, Albert, 1643. Medicinam rite discendi et exercendi methodus. Leiden: de Vogel.

Lecocq, Pascal, 1590. Bibliotheca medica, sive catalogus illorum, qui ex professo artem medicam in hunc usque annum scriptis illustrarunt. Basel: Waldkirch.

Locke, John, 1686. Méthode nouvelle de dresser des recueils. Bibliothèque universelle et historique, $2,315-340$

Maclean, Ian, 2002. Logic, Signs and Nature in the Renaissance. The Case of Learned Medicine. Cambridge: Cambridge University Press.

Melanchthon, Philipp, 1521. Loci communes rerum theologicarum seu hypotyposes theologicae. Wittenberg: [ohne Druckervermerk].

Meinel, Christoph, 1995. Enzyklopädie der Welt und Verzettelung des Wissens: Aporien der Empirie bei Joachim Jungius. In: Franz Eybl, Wolfgang Harms und Hans-Henrik Krummacher, Hg., Enzyklopädien der frühen Neuzeit: Beiträge zu ihrer Erforschung. Tübingen: Niemeyer, 162-187.

Meynell, G. G., 1993. John Locke's Method of Commonplacing, as Seen in His Drafts and His Medical Notebooks, Bodleian MSS Locke d. 9, f. 21 and f. 23. Seventeenth Century, 8, $245-267$.

Milton, J. R., 1987. John Locke's Adversaria. The Locke Newsletter, 18, 63-74.

Milton, J. R., 1997. John Locke's Medical Notebooks. The Locke Newsletter, 28, 135-156.

Morhof, Daniel Gerhard, 1692. Tractatus polyhistoricus de excerpendi ratione. Lübeck: Böckmann.

Moss, Ann, 1996. Printed Commonplace-Books and the Structuring of Renaissance Thought. Oxford: Clarendon Press.

Moss, Ann, 2011. Power and Persuasion. Commonplace Culture in Early Modern Europe. In: David Cowling und Mette Bruun, Hg., Commonplace Culture in Western Europe in the Early Modern Period. Leuven/Paris/Walpole, MA: Peeters, 1-17.

Mundella, Aloysius, 1568 Theatrum Galeni, hoc est, universae medicinae a medicorum principe Galeno diffuse sparsimque traditae promptuarium. Quo vel indicis loco in omnes Galeni libros, vel locorum communium instar in re medica, lector eruditus magna commoditate, nec minori facilitate utetur. Basel: per Eusebium Episcopium et Nicolai Fratris haeredes.

Ong, Walter, 1958. Ramus, Method, and the Decay of Dialogue. From the Art of Discourse to the Art of Reason. Cambridge, MA: Harvard University Press.

Placcius, Vincent, 1689. De arte excerpendi. Vom belahrten Buchhalten Liber singularis. Stockholm/Hamburg: Liebezeit.

Pomata, Gianna, 2010. Sharing Cases: The Observationes in Early Modern Medicine. Early Science and Medicine, 15, 193-236.

Pomata, Gianna, 2011. A Word of the Empirics: The Ancient Concept of Observation and Its Recovery in Early Modern Medicine. Annals of Science, 65, 1-25.

Pomata, Gianna/Siraisi, Nancy G., Hg., 2005. Historia. Empiricism and Erudition in Early Modern Europe. Cambridge, MA: MIT Press. 
Sacchini, Francesco, 1614. De ratione libros cum profectu legendi. Ingolstadt: apud E. Angermariam.

Schenck von Gräfenberg, Johannes, 1594. Observationum medicarum rariorum liber [...] II. Freiburg: Böckler.

Schmidt-Biggemann, Wilhelm, 1983. Topica universalis. Eine Modellgeschichte humanistischer und barocker Wissenschaft. Hamburg: Meiner.

Seidel, Bruno, 1589. Paroemiae ethicae sive sententiae proverbiales et morales, versibus antiquis conscriptae, et rhythmis Germanicis donatae [...]. Adiecto indice locorum communium copiosissimo. Frankfurt: Bassaeus.

Senfelder, Leopold, 1901. Georg Handsch von Limus. Lebensbild eines Arztes aus dem XVI. Jahrhundert. Wiener klinische Rundschau, 495-499, 514-516, 533-535.

Sharpe, Kevin, 2000. The Politics of Reading in Early Modern England. New Haven: Yale University Press.

Smolka, Josef/Vaculínová, Marta, 2010. Renesanční lékař Georg Handsch (1529-1578). DVT Dějiny věd a techniky, 43, 1-26.

Sole, Brunoro a/Schultes, Jacob 1607 Loci communes juris caesarei, pontificii et saxonici. Opus legentibus, consulentibus, judicibus, advocatis utile, facile, necessarium. Leipzig: Grosius.

Spachius, Israel, 1591. Nomenclator scriptorum medicorum. Hoc est: Elenchus eorum, qui artem medicam suis scriptis illustrarunt, secundum locos communes ipsius medicinae; cum duplici indice et rerum \& authorum. Frankfurt: Bassaeus.

Stolberg, Michael, 2003. Homo patiens. Krankheits- und Körpererfahrung in der Frühen Neuzeit. Weimar: Böhlau.

Stolberg, Michael, 2007. Formen und Funktionen ärztlicher Fallbeobachtungen in der Frühen Neuzeit (1500-1800). In: Johannes Süßmann, Susanne Scholz und Gisela Engel, Hg., Fallstudien: Theorie - Geschichte - Methode. Berlin: Trafo-Verlag, 81-95.

Valleriola, Francisco, 1562. Loci medicinae communes, tribus libris digesti. Lyon: apud haeredes S. Gryphii.

Yeo, Richard, 2004. John Locke's New Method of Commonplacing. Managing Memory and Information. Eighteenth-Century Thought, 2, 1-38.

Zedelmaier, Helmut/Mulsow, Martin (Hg.), 2001. Die Praktiken der Gelehrsamkeit in der Frühen Neuzeit. Tübingen: Niemeyer.

Zedlitz, Kathrin, 2010. Pica. Die Geschichte einer vergessenen Essstörung. Duisburg: WiKu-Verlag.

Michael Stolberg

Institut für Geschichte der Medizin

Universität Würzburg

Oberer Neubergweg 10a

97074 Würzburg

Deutschland

E-Mail: michael.stolberg@uni-wuerzburg.de 